\title{
THERMAL DILATION MONITORING OF COMPLEX URBAN INFRASTRUCTURE USING HIGH RESOLUTION SAR DATA
}

\author{
Kanika Goel $^{(1)}$, Fernando Rodriguez Gonzalez $^{(1)}$, Nico Adam $^{(1)}$, Javier Duro $^{(2)}{ }^{\text {, Marc Gaset }}{ }^{(2)}$
}

(1) Remote Sensing Technology Institute (IMF), German Aerospace Center (DLR), Germany

(2) Altamira Information, Spain

\begin{abstract}
Monitoring the deformation of urban infrastructure is crucial for improving safety and reducing economic loss. Ground deformation (caused by construction, groundwater extraction etc.) can be measured with millimeter accuracy using advanced stacking techniques in differential SAR interferometry. However, deformation might also occur due to thermal dilation of complex urban objects such as buildings and bridges, resulting in structural stress and thus, a need for monitoring. High resolution SAR missions e.g. TerraSAR-X provide a unique opportunity to measure such small seasonal displacements. This paper investigates different techniques such as Persistent Scatterer Interferometry (PSI), Distributed Scatterer Interferometry (DSI), Stable Point Network (SPN) and Small Baseline Subset Algorithm (SBAS) for thermal dilation monitoring of complex urban infrastructure using Xband SAR data.
\end{abstract}

Index Terms - Distributed Scatterer (DS), Deformation, Differential Interferometric SAR (DInSAR), Persistent Scatterer (PS), TerraSAR-X, Thermal Dilation, Urban Areas.

\section{INTRODUCTION}

Differential Interferometric SAR (DInSAR) and multitemporal DInSAR have proven to be very effective in mapping surface displacement [1]. The TerraSAR-X mission provides meterresolution imagery data from space and has provided a significant improvement in the monitoring capabilities of interferometric techniques [2]. Due to reduced wavelength (ca. $3.1 \mathrm{~cm}$ ), the data are more sensitive to small displacements, even those caused by thermal dilation of objects. However, most of the stacking algorithms, such as Persistent Scatterer Interferometry (PSI), estimate the deformation time series assuming a linear subsidence [3]. This is especially not true in the case of man-made structures, such as steel core bridges and specific buildings, which may be very sensitive to thermal dilation effects correlated to the temperature and may undergo large non-linear seasonal deformation. Thus, assumption of a linear model may often lead to ambiguous velocity estimates and decreased performance. Thus, a DInSAR stacking system dealing with non-linear motion has to be adapted accordingly.

The aim of this paper is thermal dilation monitoring of complex urban structures at high resolution by extending standard multitemporal DInSAR techniques based on
Persistent Scatterers (PSs) and/or Distributed Scatterers (DSs) [3]-[7]. Focus is on the preservation of single buildings and transportation infrastructure such as bridges, tunnels and railways. The advanced processing system is demonstrated over the urban area of Cologne, Germany using high resolution spotlight TerraSAR-X data.

\section{METHODOLOGY}

The coherent stacking methods involve the simultaneous processing of a stack of SAR images of the same area, which are taken at different times, for monitoring deformation time series and residual topography (after compensation of a digital elevation model).

In PSI, long time stable PSs are exploited and differential interferograms with respect to a single master image are formed [3]. It provides a parametric estimation of the $3 \mathrm{D}$ location and displacement for PSs based on the assumption of one or two dominant scatterers in the resolution cell. Basically, the differential interferometric phase is composed of phase components due to deformation, residual topography, atmosphere and noise. The deformation phase for a scatterer with respect to a reference pixel is usually assumed to follow a constant velocity model and is given by:

$$
\phi_{\text {defo }}^{k}=\frac{4 \pi}{\lambda}\left[v B_{t}^{k}\right]
$$

where $\lambda$ is the signal wavelength, $v$ is the displacement velocity of the scatterer in the line-of-sight (LOS) and $B_{t}^{k}$ is the temporal separation (i.e. temporal baseline) for $k$ th interferogram. The unknown deformation parameter to be estimated in this case is $v$. This model can be extended to include periodic displacement induced by thermal dilation effects associated to temperature. The yearly temperature profile of an area can be approximated by a cosine function and the extended deformation model is given as follows:

$$
\phi_{\text {defo }}^{k}=\frac{4 \pi}{\lambda}\left[v B_{t}^{k}+a\left\{\begin{array}{l}
\cos \left(\frac{2 \pi}{T}\left(B_{t}^{k}+t_{r e f}\right)\right) \\
-\cos \left(\frac{2 \pi}{T} t_{r e f}\right)
\end{array}\right\}\right]
$$

where $a[\mathrm{~mm}]$ is the amplitude of seasonal motion of the scatterer in radar's LOS, $T$ is the period of the seasonal 
displacement and is equal to one year for seasonal deformation caused by thermal dilation and $t_{r e f}$ is the time offset of the master (reference) acquisition. The unknown deformation parameters to be estimated now include $v$ and $a$.

Additionally, it is also possible to exploit partially coherent DSs via techniques such as Distributed Scatterer Interferometry (DSI), Stable Point Network (SPN) and Small Baseline Subset Algorithm (SBAS). DSs also make up a significant percentage in X-band and considerably increase the measured points, as shown later in the next section.

In $\boldsymbol{D S I}$, first, an adaptive spatial phase filtering is performed on coherent DSs [4]. The linear and seasonal deformation is then retrieved for each selected DS by adjusting the extended deformation model to the available data, similar to PSI. In cities, differential interferograms with respect to a single master image can be used, since, DSs provide a long time coherent radar return on man-made structures (however, for other applications in non-urban areas, it is better to use smallbaseline interferograms so as to limit the effects of temporal decorrelation).

The $\boldsymbol{S P N}$ technique [5], [6] also utilizes differential interferograms formed with respect to a single master image and then multilooked. Since the temperature evolution during the year does not follow pure cosine behavior, this algorithm correlates the thermal component with the air temperature history at the acquisition times as follows:

$$
\phi_{\text {defo }}^{k}=\frac{4 \pi}{\lambda}\left[v B_{t}^{k}+a_{\text {temp }} \Delta_{\text {temp }}\right]
$$

where $a_{\text {temp }}\left[\mathrm{mm} /{ }^{\circ} \mathrm{C}\right]$ is the thermal amplitude of the scatterer in radar's LOS and $\Delta_{\text {temp }}$ is the normalized temperature difference between the two acquisition times. The unknown deformation parameters to be estimated are $v$ and $a_{\text {temp }}$.

$\boldsymbol{S} \boldsymbol{B} \boldsymbol{A} \boldsymbol{S}$, meanwhile, uses unwrapped and multi-looked smallbaseline differential interferograms and estimates non-linear deformation of the scatterers without any modelling via the Singular Value Decomposition method [7]. Thus, the estimated deformation time series include the thermal dilation component of motion.

\section{EXPERIMENTAL RESULTS}

The test site is the city of Cologne in Germany. The data comprises of 50 High Resolution Spotlight TerraSAR-X images from March, 2009 to December, 2010.

Fig. 1 shows the estimated amplitude of seasonal deformation for the test site using PSI. The following different types of urban infrastructure have been highlighted:

\subsection{Buildings}

The important buildings which have undergone seasonal motion have been enclosed in white rectangles. Building ' $A$ ' is the shopping center 'Koeln Arcaden', ' $\mathrm{B}$ ' is the international trade fair and exhibition center 'Koelnmesse', ' $\mathrm{C}$ ' is the indoor concert arena 'Lanxess Arena', 'D' is Cologne's main railway station 'Koeln Hauptbahnhof', ' $E$ ' is the 'Cologne Cathedral' and is a World Heritage Site, and ' $F$ ' is an urban regeneration neighborhood 'Media Park' which houses companies, cultural institutions, a hotel and apartments. Using TerraSAR-X data, thermal dilation of these buildings is measurable.

\subsection{Bridges}

The bridges over river Rhine have been marked by yellow arrows. Bridge ' $\mathrm{G}$ ' is the 'Hohenzollernbruecke', the most heavily used railway bridge in Germany. Bridge ' $\mathrm{H}$ ' is the 'Zoobruecke' and it further extends to both east and west of the river. It is the world's most tensioned box girder bridge and also the busiest bridge in Cologne. Bridge ' $\mathrm{I}$ ' is the 'Muelheimerbruecke'. Strong periodic deformation can be seen on the bridges, since, temperatures in Cologne can vary greatly between summer and winter. However, for some parts of the bridges, measurement point density is less. Possible reasons for this include river-bridge multiple bounce, double scatterers and non-linear displacements not related to temperature.

\subsection{Railways}

The 'Line 13' of the Cologne Stadtbahn, a light rail system, has been enclosed in the red rectangle ' $J$ '. The northern part of this line is built as an elevated railway and the seasonal motion is evident.

We have zoomed-in on the 'Zoobruecke' bridge in Fig. 2. The left image shows the estimated amplitudes of seasonal deformation using PSI. The area enclosing the 'Zoobruecke' bridge has also been processed using the $\boldsymbol{D S I}$ algorithm and the results are shown in the image on the right. The results for PSs and DSs match well with each other. More importantly, there is a substantial increase in the measured point density.

Another example illustrating 'Hohenzollernbruecke' and 'Duetzer Bruecke' (not shown previously in Fig. 1) bridges is provided in Fig. 3. It shows the estimated thermal amplitudes for the test area using $\boldsymbol{S P N}$. Local temperature data has been used instead of a cosine modelling. It is worth mentioning that the use of local registered temperatures instead of a cosine model is sometimes not an optimal solution because it can be difficult to get data from a station near the area of interest for specific SAR acquisition times. Additionally, the real temperature of each structure also depends on the solar irradiation, the materials's absorbed solar radiation, temperature gradients and surrounding elements which locally affect the temperature suach as rivers or sea. Thus, the registered temperature can differ several degrees from the real temperature of the structure. The unmodeled thermal signal produces a residual in the long-term displacement measurement and a decrease in the model fitting quality.

Note that in all these three methods demonstrated previously, wherein, a deformation model is fitted to the available phase data, it is suggested to have at least two years of acquisitions for measuring thermal dilation as this improves the model fitting.

Lastly, Fig. 4 shows the mean deformation estimated using the $\boldsymbol{S B} \boldsymbol{A} \boldsymbol{S}$ technique. The area near 'Lanxess Arena' building and 'Zoobruecke' bridge is shown. Deformation time series is visualized for a point on the concert arena building. As can be 
seen, even non-linear deformation time series can be estimated, which includes the linear as well as seasonal deformation due to thermal dilation. However, SBAS is susceptible to phase unwrapping errors in urban areas due to layover, shadow and sometimes, water bodies dividing the SAR scene into two (as is the case for our test site Cologne). Sparse phase unwrapping should be used which only unwraps the coherent points.

\section{CONCLUSION}

The results demonstrate the potential of TerraSAR-X High Resolution Spotlight data to measure the thermal dilation of complex structures such as bridges. Different techniques such as PSI, DSI, SPN and SBAS have been discussed for this purpose. It is now possible to monitor the thermal stress of urban infrastructure for stability analysis.

\section{REFERENCES}

[1] R. Bamler and P. Hartl, "Synthetic aperture radar interferometryTopical review," Inverse problems- 14, R1 - R54, IOP Publishing Limited (UK), 1998.

[2] N. Adam, M. Eineder, N. Yague-Martinez, and R. Bamler, "High resolution interferometric stacking with TerraSAR-X," In: Proc. IGARSS, Boston, US, 2008.

[3] A. Ferretti, C. Prati, and F. Rocca, "Permanent scatterers in SAR interferometry,” IEEE TGARS, vol. 39, No. 1, pp. 8-20, 2001.

[4] K. Goel and N. Adam, "High resolution differential interferometric stacking via adaptive spatial phase filtering," In: Proc. IGARSS, Vancouver, Canada, 2011.

[5] J. Duro, J. Inglada, J. Closa, N. Adam, and A. Arnaud, "High Resolution Differential Interferometry using time series of ERS and ENVISAT SAR data," In: Proc. FRINGE, Frascati, Italy, 2003.

[6] J. Duro, O. Mora, M. Agudo, and A. Arnaud, "First results of Stable Point Network software using TerraSAR-X data," In: Proc. EUSAR, Aachen, Germany, 2010.

[7] P. Berardino, G. Fornaro, R. Lanari, and E. Sansosti, "A new algorithm for surface deformation monitoring based on small baseline differential SAR interferograms," IEEE TGARS, vol. 40, no. 11, pp. 2375-2383, 2002.

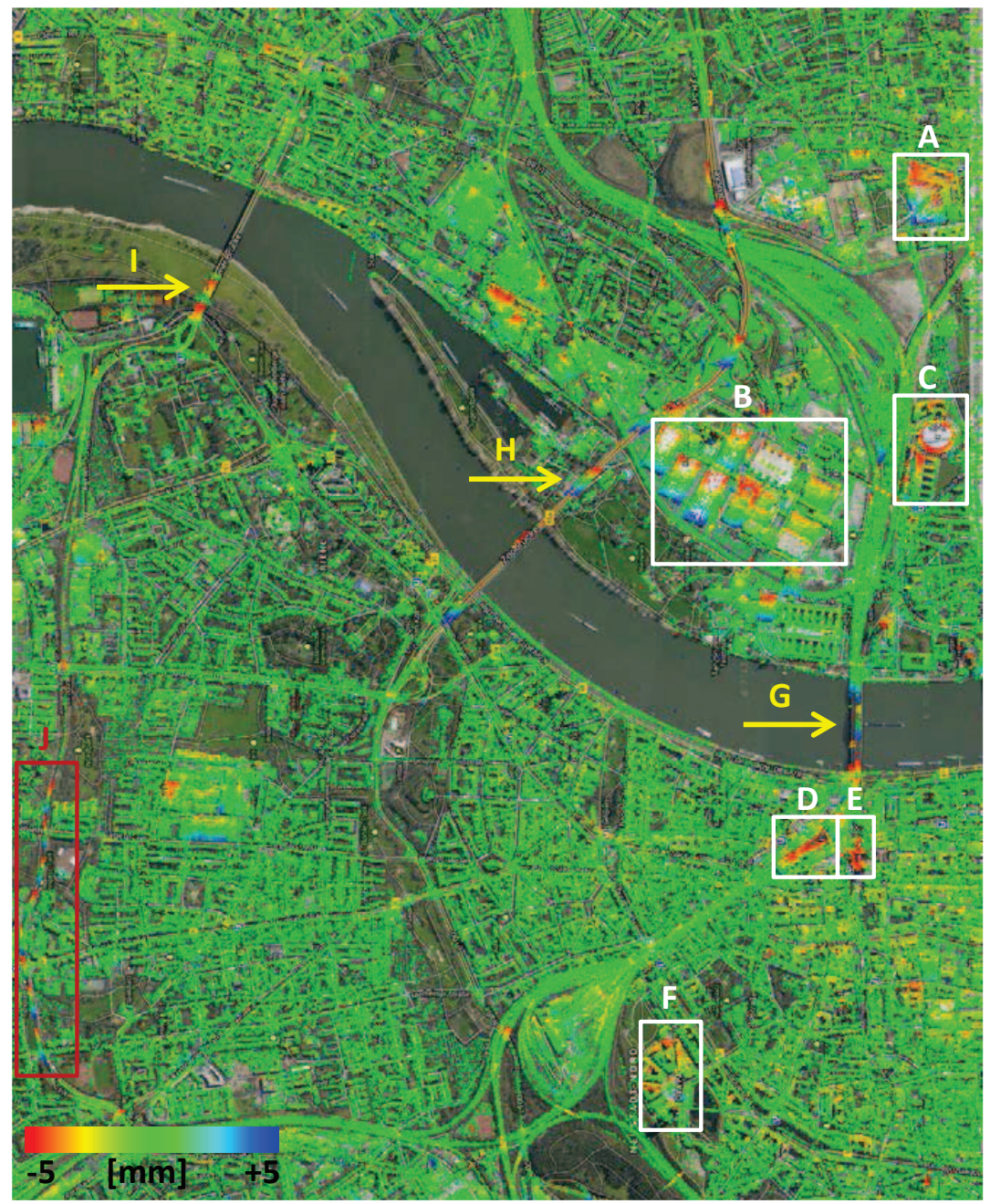

Fig. 1. Estimates of amplitude of seasonal motion (caused by thermal dilation) for Cologne, Germany, via PSI exploiting TerraSAR-X data from March, 2009 - December, 2010. Different types of urban structures have been highlighted in white (buildings), yellow (bridges) and red (railways). 

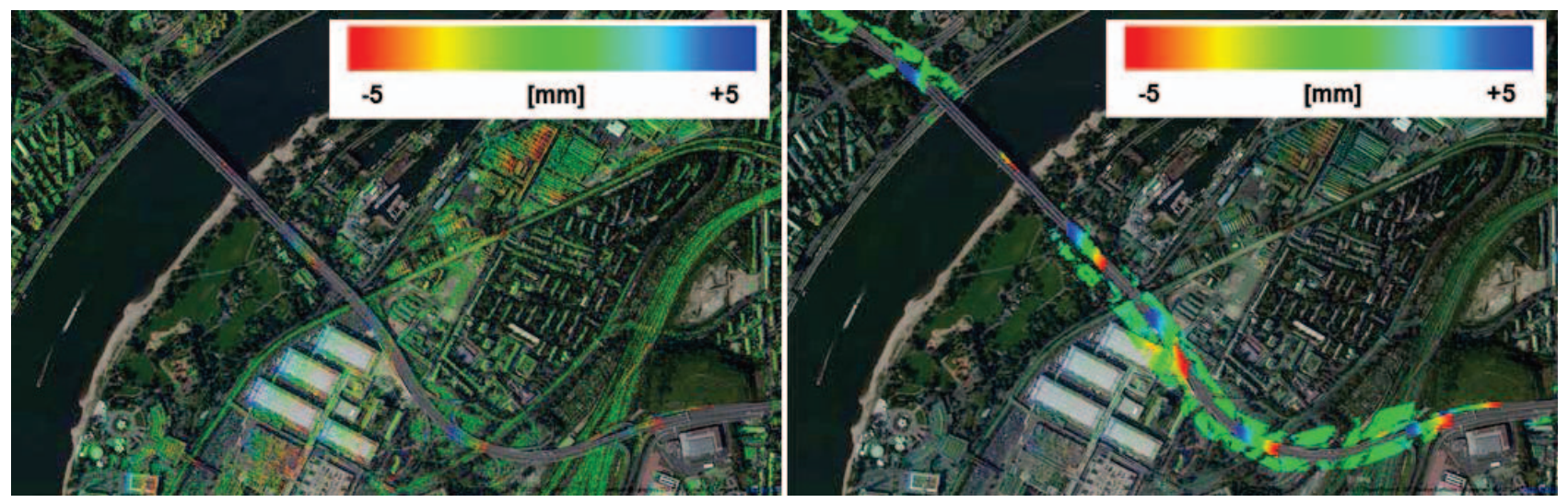

Fig. 2. Estimates of amplitude of seasonal motion (caused by thermal dilation) for 'Zoobruecke' bridge in Cologne, Germany, via PSI (left image) and DSI (right image), respectively, exploiting TerraSAR-X data from March, 2009 - December, 2010. It can be observed that DSI provides a high density of measured points as compared to PSI.

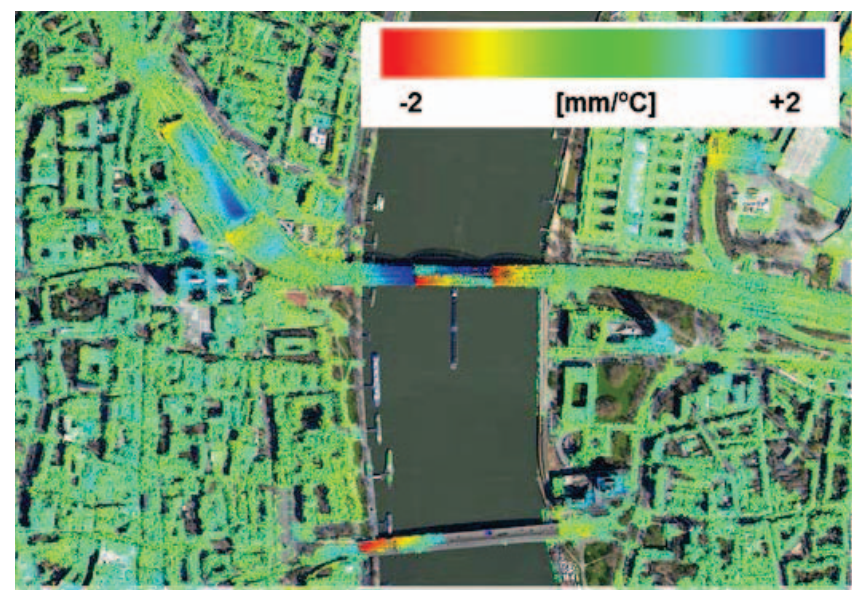

Fig. 3. Estimates of thermal amplitudes for Hohenzollernbruecke' (top) and 'Duetzer Bruecke' (bottom) bridges in Cologne, Germany, via SPN technique exploiting TerraSAR-X data from March, 2009 - December, 2010. Local temperature data was used for the processing instead of a cosine modelling.
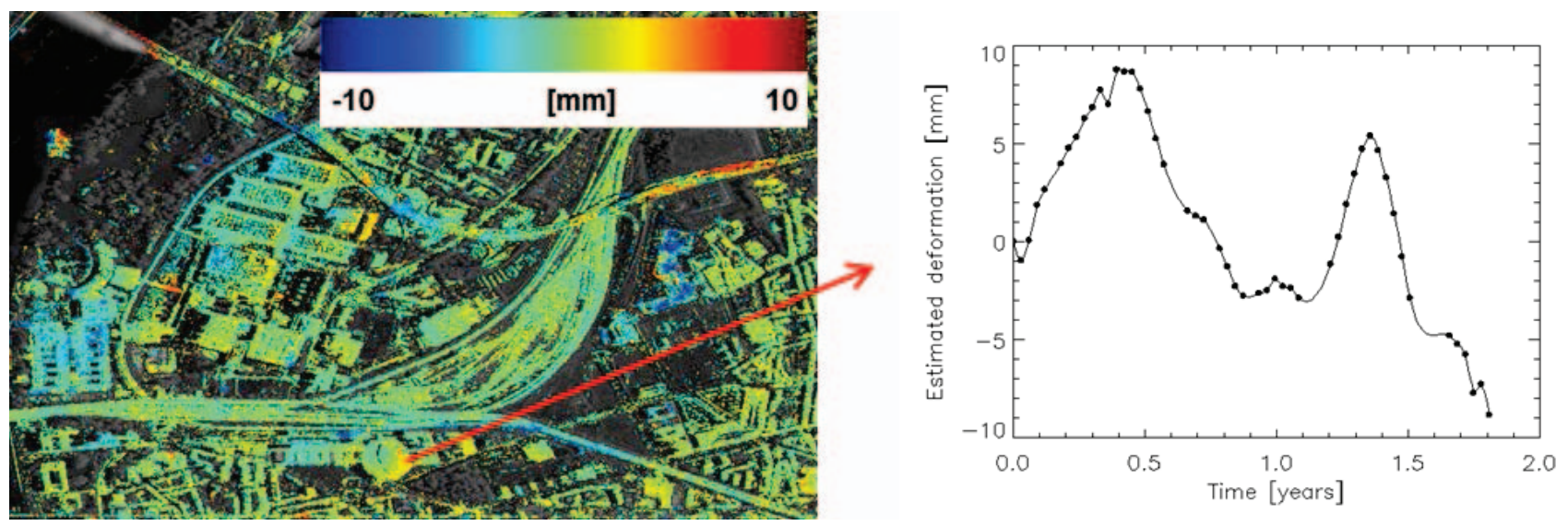

Fig. 4. Mean deformation estimates for area near 'Lanxess Arena' building and 'Zoobruecke' bridge in Cologne, Germany, via SBAS exploiting TerraSAR-X data from March, 2009 - December, 2010. Non-linear deformation time series is visualized for a point on the concert arena building, which includes linear as well as seasonal deformation due to thermal dilation. 